\title{
Un proyecto integrado para la formación de los futuros maestros: elaboración de páginas web para la enseñanza de la literatura en educación infantil y primaria ${ }^{1}$
}

An Integrated Approach for the Training of Students in Education Degrees: Website creation to Teach Literature in Infant and Primary Education

\author{
Francesc Rodrigo-SeGura ${ }^{2 *}$ \\ frodrigo@florida-uni.es, http://orcid.org/0000-0003-3937-9946 \\ JOSEP BALLESTER-ROCA** \\ josep.ballester@uv.es, https://orcid.org/0000-0002-1995-3253 \\ *Florida Universitària, Catarroja-València, España \\ **Universitat de València, València, España \\ Grupo de Investigación Educación Lectora, Literaria, Cultura y Sociedad (ELCIS)
}

\section{Resumen:}

El objetivo de esta investigación es reflexionar sobre la idoneidad del paradigma de la educación literaria como marco de referencia para la elaboración de propuestas didácticas en los grados de Magisterio, basadas en la metodología de secuencias didácticas o proyectos para la formación lectora y literaria de los estudiantes. Una

\begin{abstract}
:
The aim of this article is to reflect on the pertinence of the paradigm of literary education as a framework for the elaboration of teaching proposals in Infant and Primary Education Degrees. This type of training contributes to both developing students' communicative competence, while also raising in them an understanding of
\end{abstract}

1 El presente trabajo se enmarca en el proyecto "Educación lectora, literaria, mediática, digital, diversidad(es) y ciudadanía" (UV-SFPIE_GER17-589447), concedido en el marco de la convocatoria de innovación del Vicerrectorado de Políticas de Formación y Calidad Educativa de la Universitat de València.

2 Dirección para correspondencia (correspondence address):

Francesc Rodrigo Segura. FLORIDA UNIVERSITÀRIA (Unidad de Educación). C/ Rei en Jaume I, 2. C.P. 46470 Catarroja, Valencia (España). 
Un proyecto integrado para la formación de los futuros maestros: elaboración de páginas web para la enseñanza de la literatura en educación infantil y primaria

Francesc Rodrigo-Segura y Josep Ballester-Roca

formación que contribuye a desarrollar su competencia comunicativa y a considerar la lectura como un aspecto básico en la vida de las personas como fuente de placer, aprendizaje, creatividad y enriquecimiento personal. Para ello se presenta una experiencia de trabajo por proyectos realizada en $2^{\circ}$ curso consistente en la creación de páginas web con recursos para la formación literaria del alumnado de Educación Infantil y Primaria.

El estudio se ha lleva a cabo a partir de una metodología mixta, combinando técnicas cuantitativas y cualitativas, basada en la investigación-acción en las aulas para comprobar si esta opción metodológica resulta, también, en estudios superiores, una herramienta eficaz para el aprendizaje que contribuye a realizar una enseñanza competencial, integrando en los proyectos las competencias específicas de las materias de Formación Literaria y las competencias transversales los grados universitarios (trabajo en equipo, innovación, creatividad, uso de las TIC, etc.). Y si, además, ofrece posibilidades que potencien el trabajo interdisciplinar y colaborativo del alumnado y del profesorado. En definitiva, se intentará validar la metodología de proyectos de educación literaria como una propuesta pedagógica útil para alcanzar los objetivos que la sociedad actual demanda a la formación literaria de los futuros maestros y maestras.

\section{Palabras clave:}

Educación literaria; Trabajo por proyectos; Formación en competencias; Competencia TIC; Grados de Magisterio; Estudios Superiores. reading as a basic aspect of people's lives which is productive of pleasure, learning, creativity and personal enrichment. More specifically, this article reports on a project undertaken in the second year of both degrees, which consisted of the design of webpages as a tool for promoting Infant and Primary Education students' literacy. We used a mixed methodology which encompassed quantitative and qualitative techniques, based on action research, with a view to exploring (i) whether this methodological option proved to be an effective tool for inducing students' learning based on the acquisition of skills and transversal competencies (teamwork, innovation, creativity, use of ICT, etc.); and (ii) whether the project gives opportunities for interdisciplinary and collaborative work between teachers and students. Literary education projects are a useful methodology to achieve the objectives that current society requires from future teachers.

\section{Key words:}

Literary education; Project Based Learning; Training in skills; ICT competence; Teacher training; Higher education.

\section{Résumé:}

L'objectif de cette recherche est de réfléchir sur la pertinence du paradigme de l'éducation littéraire en tant que cadre de référence pour l'élaboration de propositions didactiques dans les degrés de l'enseignement, sur la base de la méthodologie des séquences didactiques ou des projets de lecture et de formation littéraire des étudiants. Une formation qui contribue à développer leur compétence communicative et à considérer la lecture comme un aspect fondamental dans la vie des gens, comme source de plaisir, d'apprentissage, de créativité et d'enrichissement personnel. Pour cela, une expérience de travail par des projets réalisés en 2ème année est présentée, consistant à créer des pages Web avec des ressources pour la formation littéraire des étudiants en enseignement primaire et secondaire.

En utilisant une méthodologie qualitative basée sur la recherche-action dans les salles de classe, cette expérience sera analysée pour démontrer que, dans les études supérieures, 
Un proyecto integrado para la formación de los futuros maestros: elaboración de páginas web para la enseñanza de la literatura en educación infantil y primaria

Francesc Rodrigo-Segura y Josep Ballester-Roca

c'est aussi un outil efficace d'apprentissage, puisqu'il place la figure du lecteur (l'élève) en tant que principal protagoniste de l'événement littéraire, contribue à un apprentissage par compétences, en intégrant dans les projets les compétences spécifiques des matières de la formation littéraire et des compétences transversales aux diplômes universitaires (travail d'équipe, innovation, créativité, utilisation des TIC, etc.) et offrant, en outre, de grandes possibilités de travail interdisciplinaire et collaboratif.

Pour résumer, nous essaierons de valider la méthodologie des projets d'éducation littéraire en tant que proposition pédagogique utile pour atteindre les objectifs que la société exige aujourd'hui de la formation littéraire des futurs enseignants.

\section{Mots clés:}

Éducation littéraire; Travail par projets; Formation en compétences; Compétence en TIC; Degrés d'enseignement; Études supérieures.

Fecha de recepción: 15-7-2018

Fecha de aceptación: 18-10-2018

\section{Introducción}

En este artículo se presenta una experiencia realizada en los grados de Magisterio de Florida Universitària (centro adscrito a la Universitat de València) que ha implantado un modelo educativo basado en la metodología de Proyectos Integrados ${ }^{3}$ para desarrollar las competencias profesionales y conseguir un aprendizaje pertinente ajustado a las necesidades reales de los estudiantes y a las exigencias de la sociedad actual. Para ello se han diseñado propuestas que favorecen un cambio en el proceso de enseñanza-aprendizaje que permita enlazar la reflexión teórica con la práctica, potenciar la innovación, a través del trabajo colaborativo del alumnado y del profesorado, y ofrecer experiencias de aprendizaje verdaderamente significativas. En concreto, se describe la experiencia realizada en las asignaturas de Formación literaria en el marco del Proyecto Integrado global de segundo curso titulado: Diversidad y recursos educativos.

3 Para profundizar en el modelo educativo de Florida, basado en el desarrollo de las competencias profesionales mediante Proyectos Integrados, se puede consultar la comunicación: Desenvolupament d'un model educatiu centrat en les competències professionals i el treball per projectes en els graus d'educació de Florida Universitària. Actas del Congreso Internacional de Docencia Universitaria e Innovación (CIDUI), (2012), Barcelona: Universitat Pompeu Fabra. 


\section{Marco teórico}

La siguiente investigación se inserta en el paradigma teórico de la educación literaria y en su aplicación didáctica mediante la metodología de proyectos integrados en los estudios superiores, en concreto, en los Grados de Maestro/a de Educación Infantil y Primaria. Para ello partimos de las reflexiones realizadas por diversos investigadores sobre la importancia que la educación literaria tiene en la formación de los futuros maestros: Mendoza (1998, 2004), Ballester (1999, 2007, 2015); Colomer (2002); Cerrillo (1990, 2007); Ballester e Ibarra (2013 a, b, 2016). De estas propuestas se desprenden cuatro grandes objetivos de la formación literaria en estos estudios:

1. Obtener las claves de acceso a la literatura con un lenguaje mediante el cual se expresa la experiencia humana -en todos sus órdenes: imaginativo, moral, vivencial- y que es una de las vías que el niño tiene de comprensión del mundo y de anexión de toda experiencia y sensibilidades personales.

2. Dar a conocer el medio y la tradición cultural propios; aspecto especialmente relevante en la coyuntura histórica actual, pues, contribuye, desde la escuela, al proceso de identificación cultural colectiva que vive la sociedad.

3. Hacer que el conocimiento del registro literario de la lengua revierta en la capacidad expresiva de los niños y jóvenes.

4. Plantear la lectura como comprensión de unos códigos artísticos convencionales y, respecto al conocimiento de la naturaleza específica de la obra literaria, se tiene que educar en el placer estético.

Para desarrollar estos objetivos contamos con un marco ya establecido, el paradigma de la educación literaria, que ofrece los elementos necesarios para intentar una efectiva competencia literaria en las primeras etapas escolares y en la educación secundaria. Sin embargo, en estudios superiores lo que hace falta es, tal y como apunta Colomer $(2002,16)$ : "saber qué competencias tienen que adquirir los alumnos y qué prácticas ayudan a su desarrollo, para poder incluirlas y abordarlas en los planes de formación de los futuros maestros". Además, hay que tener en cuenta, como propone Ballester $(2007,2015)$, que la formación profesional de los docentes debe atender a tres aspectos básicos: considerar con atención la adecuación de su preparación pedagógica general al campo 
específico de la literatura, revisar críticamente las programaciones que se llevan a cabo en las aulas y buscar el modelo de formación más conveniente para que, en el aula, se pueda enseñar literatura en función de estos objetivos concretos. Para ello desde el modelo de educación literaria se formulan diversas estrategias para conseguir alcanzar las competencias y los objetivos que acabamos de definir:

- La lectura por placer: para conseguir que el estudiante pueda adquirir un conocimiento empírico de los géneros y tipologías de textos literarios en toda su gama y diversidad.

- La lectura técnica: a través del conocimiento de la teoría literaria y la aplicación de métodos de comentario y análisis de textos para adquirir la formación conveniente y conseguir unos instrumentos mínimos de crítica literaria.

- El conocimiento teórico y práctico de la historia literaria a través de sus textos y obras.

- La capacitación de los estudiantes para el análisis de textos y su transformación en propuestas didácticas adecuadas para los diversos niveles de enseñanza.

Otro elemento esencial a tener en cuenta en la formulación del proyecto de formación literaria que se describe en estas páginas es que éste, tal y como se ha explicado en la introducción, se enmarca en un proyecto global de carácter transdisciplinar titulado: Diversidad y recursos educativos, en el cual participan todas las asignaturas de $2^{\circ}$ curso del grado. Los referentes pedagógicos en los que se sustenta esta propuesta son el pensamiento complejo (Morin, 1999, 2006) y la pedagogía colectiva (Deleuze y Guattari, 1994, 2001), que plantean el diseño de nuevas experiencias de aula orientadas a enlazar conocimientos y vivencias que trasciendan el espacio disciplinar. Por ello los aprendizajes se entretejen para dar respuesta a la nueva racionalidad compleja, a través de la transdisciplinariedad, mediante la confluencia de las disciplinas que derivan en conocimientos producto de estas interacciones y de la complementariedad entre conocimiento y realidad. Se trata de unir aprendizajes, conocimientos, experiencias y vivencias, desde el ser, el hacer, el conocer y el convivir, (Delors, 1996). Este aprendizaje rizomático (Deleuze y Guattari, 1994, 2001) permite la individualización, el intercambio de saberes y favorece la articulación de sistemas educativos abiertos que enlazan el conocimiento, la profesionalización y la vida. La pedagogía 
colectiva se basa en la práctica colaborativa y en el aprendizaje como proceso compartido y comunitario.

Este es el marco teórico y pedagógico en el que se sitúa la experiencia realizada y que está basada en la metodología de las secuencias didácticas o proyectos de literatura que contribuye a conseguir los objetivos y a desarrollar las estrategias del paradigma de la educación literaria, explicados en las líneas anteriores. Para justificar la adopción de esta metodología nos basamos en las aportaciones de Margallo (2005, 2012 a, b) que defiende que la metodología de proyectos de literatura es una de las formas de programación que mejor se adapta al modelo de educación literaria puesto que tiene como objetivo la formación del lector literario. Las razones que apunta son las siguientes:

En primer lugar, la autora subraya que los proyectos de trabajo de literatura se relacionan directamente con los objetivos que mejor contribuyen a la formación de lectores literarios: familiarizarse con el circuito social del libro, establecer vínculos personales con la literatura, apreciar lecturas diversas, dominar las habilidades de interpretación y utilizar la información contextual en la comprensión del texto, tal y como señala Colomer $(2005,2012)$. Además los proyectos literarios potencian las dos dimensiones de la formación de lectores: consolidar los hábitos de lectura y avanzar en la capacidad en la interpretación de los textos mediante el acceso a una amplia variedad de textos, la capacidad de utilizar un lenguaje metaliterario y la activación de la información contextual para comprender el texto. Otro elemento destacable de esta metodología es que, en contraste con la atomización de contenidos y actividades de otros modelos de programación, en los proyectos los contenidos se insertan en un itinerario necesario para la elaboración del producto final dotando así de intencionalidad al aprendizaje literario.

Por último, de la evolución de los proyectos de lengua o literatura en la actualidad cabe destacar la importancia de la utilización de las TIC, puesto que, según diversos autores (Zayas, 2011; Margallo, 2012b; Manresa, Duran y Ramada, 2012) su incorporación comporta importantes ventajas: potencia la proyección social de la lectura literaria, favorece la creación de vínculos entre comunidades de lectores, da auditorio a las producciones de los alumnos y proporciona instrumentos que enriquecen su diseño. 


\section{Marco metodológico}

La hipótesis de partida de esta investigación es demostrar que la metodología de proyectos de literatura es, también, en niveles universitarios, una propuesta válida para responder a los retos de la enseñanza de la literatura en la sociedad actual. Para responder a esta hipótesis se ha utilizado el método de la investigación-acción colaborativa o investigación cooperativa dentro del modelo socioconstructivista (Guba y Lincoln, 2000). Este método persigue la construcción conjunta del conocimiento y del saber ligado a la práctica educativa y resulta de la unión de las perspectivas internas (de los docentes) y de las externas (del investigador) (Desgagné, 1997). Sus bases teóricas son: la investigaciónacción (Lewin, 1946; Freire, 1975; Stenhouse, 1991; Latorre, 2003); la co-investigación (Arnal, De Rincón y Latorre, 1992) y la co-construcción del conocimiento (Desgagné, 1997).

La adopción de este método investigativo deriva de la constatación que, desde el ámbito específico de la Didáctica de la Lengua y la Literatura, el paradigma cualitativo responde al acuerdo generalizado de que la investigación en esta área no puede ser meramente descriptiva, sino que debe enfocarse hacia la práctica, hacia la comprensión y la interpretación de los procesos de enseñanza y aprendizaje (Camps, 2012 a, b). Por eso se ha utilizado una metodología de carácter crítico a través de la investigación en la acción para poder profundizar en el análisis de las prácticas. Este trabajo también tiene características del paradigma socioconstructivista, puesto que se orienta a mejorar la acción educativa en el aula a través de la construcción de un saber pedagógico y metodológico que pueda ser compartido por otros docentes. Por estas razones el objeto central de la investigación son los aspectos metodológicos, es decir, las prácticas de enseñanza y aprendizaje. Y por ello se atiende de manera prioritaria a la interrelación entre el enseñante, el aprendiz y la lengua o lenguas, objeto de enseñanza y aprendizaje, dentro de lo que se denomina el sistema didáctico (Engeström, 1987), en consonancia con las actuales líneas de investigación en el campo de la Didáctica de la Lengua y la Literatura (Camps, 2012a, b).

Recordemos que Mendoza (2011) ya señalaba en las páginas de esta revista que la investigación-acción, junto con las opciones etnográficas y la observación participante, constituyen las opciones de investigación más adecuadas para la investigación en "primeras lenguas" y en "lenguas 
extranjeras" ya que permiten que ésta emerja realmente de las mismas aulas, que constituyen "a quiet methodological revolution", en palabras de Denzin y Lincoln (citadas en Mendoza, 2001, p. 31). Cabe añadir que compartimos con Mendoza "la necesidad de reflexión crítica" puesto que:

Las modalidades de investigación aquí destacadas apuntan a la perspectiva socioconstructivista, desde la que se entiende la formación como como un continuo de procesos educativos de reflexión crítica que permiten ir construyendo un pensamiento renovado sobre el cual fundamentar actuaciones alternativas. Ello sólo es posible si se da protagonismo al docente, se valoran sus conocimientos prácticos y se revisan sus modelos interiorizados, es decir, si convergen la formación y la investigación" (Mendoza, 2011, pp. 36-37).

\section{CONTEXTO Y PARTICIPANTES EN EL PROYECTO ${ }^{4}$}

La investigación se inició en el curso 2010-11 en dos grupos de $2^{\circ}$ del Grado de Educación Infantil (84 alumnos) y tuvo continuidad en el 2011-12 con 2 grupos de infantil y 3 grupos de primaria (200 alumnos). Debido a las valoraciones positivas realizadas por el alumnado y el profesorado, desde el curso 2013-14 hasta la actualidad, el proyecto se ha institucionalizado y en su desarrollo participan todas las materias y todos los grupos de $2^{\circ}$ curso.

- Dispositivo de recogida de datos:

Los instrumentos utilizados en el ciclo de investigación-acción para dotar de validez, credibilidad y transferencia a la investigación realizada son los siguientes:

a) Cualitativos:

- Informe final del proyecto (memoria escrita y exposición oral del proyecto ante un tribunal compuesto por tres profesores del curso). Producción grupal. Se han analizado las memorias de los seis equipos del grupo de $2^{\circ} \mathrm{B}$ de infantil.

- Informe de evaluación de la asignatura: incluye cuestiones sobre la valoración del proyecto y las conclusiones sobre la asignatura

4 Datos extraídos del Trabajo Fin del Máster en Investigación en Didácticas Específicas: L'educació literària: una proposta de treball per projectes (2012) y de la Tesis Doctoral: La formació literària i la metodologia de projectes de treball en batxillerat i en els graus de mestre/a d'Educació Infantil i Primària (2016). Universidad de València. 
de formación literaria. Se han analizado las producciones individuales de 30 de los 34 estudiantes de $2^{\circ} \mathrm{B}$.

- Valoración cualitativa del Proyecto Integrado. Se han analizado las producciones de 12 estudiantes de $2^{\circ}$ curso Educación Infantil y Educación Primaria.

- Focus group con las seis coordinadoras de los equipos de $2^{\circ} \mathrm{B}$ de infantil. (Registro audiovisual).

b) Cuantitativos:

- Encuesta valoración del proyecto. Se han recogido y analizado 22 encuestas de los 34 alumnos de $2^{\circ} \mathrm{B}$.

- Resultados académicos: análisis de las notas del alumnado (comparativa de la nota final teniendo en cuenta el valor del $25 \%$ del proyecto y de la nota recalculada, sin tenerlo en cuenta). Se han analizado los resultados de 47 alumnos de $2^{\circ} \mathrm{A}$ y de 34 alumnos de $2^{\circ} \mathrm{B}$.

\section{CRITERIOS DE ANÁLIISIS: ESTABLECIMIENTO DE CATEGORÍAS}

Los datos aportados se han agrupado en las siguientes categorías:

- metodología de trabajo

- objetivos del proyecto

- aprendizajes específicos (lingüísticos y literarios)

- aprendizajes competenciales y transversales

- aprendizajes didácticos

- papel del profesorado

- papel del alumnado

- evaluación

Estas ocho categorías han sido objeto de contraste y análisis en las muestras cualitativas y cuantitativas para poder interpretar los resultados de la investigación.

\section{Experiencias didácticas y resultados}

El proyecto se desarrolla mediante las siguientes fases:

- Fase I: Explicación del proyecto y configuración de los equipos.

- Fase II: Formación con expertos. Estudio del diseño de las páginas web. 
- Fase III: Decisión sobre las aplicaciones a utilizar.

- Fase IV: Implementación de las páginas web.

- Fase V: Presentación de resultados.

\section{CONTENIDOS Y RECURSOS DE LAS PÁGINAS WEB}

Todas las páginas web comparten los mismos contenidos y recursos generados en las clase de Formación Literaria:

- Presentación: del grupo, de los objetivos y contenidos de la web, etc.

- Textos expositivos: selección de los informes, resúmenes de artículos y libros leídos, de las actividades y los trabajos de investigación realizados.

- Textos valorativos: selección de reseñas de lectura, de los textos argumentativos y de las valoraciones de las sesiones con expertos.

- Textos literarios: producciones literarias individuales o de grupo.

- Actividades y recursos generados para trabajar la educación literaria en infantil y primaria (rincones, talleres, cuentacuentos, etc.)

A continuación presentamos el listado de competencias que se desarrollan en el proyecto:

Tabla 1

Competencias específicas, comunes y resultados de aprendizaje

Competencias espe- Competencias comunes Resultados de aprendicíficas de Formación a los Grados de Educa- zaje:

Literaria

1. Conocer y utilizar de 1. Expr manera adecuada recursos para el fomento de la lectura y la escritura literaria.

2. Conocer la tradición oral y el folclore.

3. Adquirir formación li- 3. Analizar e incorporar teraria $y$, en especial, conocer la LIJ.

4. Conocer y dominar técnicas de expresión oral y escrita. por escrito en las lenguas oficiales.

2. Utilizar con solvencia 2 . las TIC como herramientas de trabajo ha- 3. Analizar críticamente bituales. de forma crítica cuestiones relevantes de la 4 . sociedad actual: multiculturalidad e interculturalidad. obras literarias y materiales curriculares

4. Valorar las competencias esenciales que niños y jóvenes adquieren a través de la LIJ. de las diversas etapas. 


\begin{tabular}{|c|c|c|}
\hline $\begin{array}{l}\text { Competencias espe- } \\
\text { cíficas de Formación } \\
\text { Literaria }\end{array}$ & $\begin{array}{l}\text { Competencias comunes } \\
\text { a los Grados de Educa- } \\
\text { ción }\end{array}$ & $\begin{array}{l}\text { Resultados de aprendi- } \\
\text { zaje: }\end{array}$ \\
\hline $\begin{array}{l}\text { 5. Conocer las fuentes } \\
\text { básicas de la investi- } \\
\text { gación en la Didácti- } \\
\text { ca de la Lengua y la } \\
\text { Literatura e identificar } \\
\text { el objeto y la metodo- } \\
\text { logía empleada. } \\
\text { 6. Analizar de manera } \\
\text { crítica materiales } \\
\text { didácticos y produc- } \\
\text { ciones literarias para } \\
\text { niños y jóvenes. }\end{array}$ & $\begin{array}{l}\text { 4. Promover el trabajo } \\
\text { cooperativo. } \\
\text { 6. Conocer y aplicar me- } \\
\text { todologías y técnicas } \\
\text { básicas de investi- } \\
\text { gación educativa y } \\
\text { ser capaz de diseñar } \\
\text { proyectos de innova- } \\
\text { ción. } \\
\text { 7. Reflexionar sobre la } \\
\text { práctica y la realidad, } \\
\text { y también contribuir } \\
\text { a la innovación y a la } \\
\text { mejora en educación. }\end{array}$ & $\begin{array}{l}\text { 5. Ser capaz de aplicar } \\
\text { en el aula estrategias } \\
\text { y métodos activos en } \\
\text { relación con la LIJ. } \\
\text { 6. Caracterizar, valorar y } \\
\text { producir varios géne- } \\
\text { ros de LIJ. } \\
\text { 8. Tener nociones de } \\
\text { investigación y de in- } \\
\text { novación en literatura } \\
\text { infantil y juvenil. }\end{array}$ \\
\hline
\end{tabular}

\section{Evaluación del Proyecto}

El Proyecto tiene un valor del $25 \%$ de la calificación de las materias. Es una nota única diferente para cada alumno/a y es la misma para todas las asignaturas que participan. Esta calificación refleja el nivel de adquisición de las competencias específicas y transversales asociadas al proyecto y se compone de la suma de la nota obtenida por el equipo y la nota individual.

Los instrumentos de evaluación son los siguientes:

- Memoria escrita del proyecto: 40\%. Evaluación realizada por el coordinador en base a los criterios de evaluación de la rúbrica de evaluación de la memoria.

- Presentación oral del proyecto: 20\%. Evaluación realizada por el coordinador y, al menos, dos profesores del grupo en base a los criterios de la rúbrica de evaluación de la comunicación oral y de la rúbrica de valoración de páginas web.

- Defensa Oral Individual: 15\%. Preguntas individuales a los miembros del equipo sobre el proyecto y valoración de la exposición individual en base a la rúbrica de comunicación oral.

- Evaluación Individual del Proceso: 25\%. Evaluación realizada por el coordinador y por el profesorado a partir de las evidencias recogidas en el desarrollo del proyecto (asistencia a formación, actas de reuniones, evaluación entre pares, participación, etc.). 


\section{Resultados de la investigación}

Respecto a los resultados cualitativos, a continuación, se presenta el análisis de las tres categorías relacionadas con los aprendizajes adquiridos, tomando como instrumento las conclusiones finales de las memorias ya que éstas ofrecen una visión bastante completa de las valoraciones de los estudiantes.

Tabla 2

Análisis de las categorías relacionadas con los aprendizajes y la evaluación del proyecto

CATEGORÍA 3: APRENDIZAJES ESPECÍFICOS (LINGÜÍSTICOS Y LITERARIOS) VALORACIÓN POSITIVA

1.- Alto grado de adquisición de los aprendizajes específicos de la asignatura de Formación Literaria: conocer las ventajas y la importancia de la LIJ para acercarnos a nuestra cultura, adquirir hábito lector, fomentar la creatividad y la imaginación, transmitir las ventajas de la literatura (enriquecimiento cognitivo, emocional y personal, desarrollar la sensibilidad y la creatividad).

2.- Se ha conocido el amplio abanico de posibilidades que nos ofrece la LIJ para acercarnos a la lectura y desarrollar la sensibilidad y la creatividad. Ha aumentado el interés personal por la lectura y se valora como fuente de placer y diversión a trabajar mediante estrategias para potenciar la creatividad y la imaginación.

3.- Se han descubierto las posibilidades variadas que ofrece el modelo de la educación literaria para contribuir a la formación del alumnado. Se considera que la literatura ha de estar presente en el mundo de los niños desde la escuela y la familia ha de continuar con la tarea de fomentar la lectura.

4.- Se valora positivamente la mejora en la expresión oral en valenciano y el desarrollo de la competencia comunicativa a través de las exposiciones orales. Además, se constata la necesidad de expresarse con corrección en valenciano con el alumnado y las familias.

CATEGORÍA 4: APRENDIZAJES COMPETENCIALES Y TRANSVERSALES

VALORACIÓN POSITIVA

1.- Todas las valoraciones coinciden en que sí que ha habido una mejora en la competencia de dominio de las TIC (importante adquirir este tipo de competencias que ayudan a aprender y a comunicarte a través de las redes).

2.- Se valora positivamente que el trabajo de las TIC, a través de una metodología innovadora, ha permitido conocer la diversidad de herramientas informáticas existentes para crear la propia web.

3.- También se han desarrollado de manera óptima las competencias de creatividad, innovación y comunicación oral (aspecto muy importante como docen tes) y se ha logrado una consideración positiva de la diversidad educativa, ésta ya no se ve como un problema sino como fuente de enriquecimiento. 
Un proyecto integrado para la formación de los futuros maestros: elaboración de páginas web para la enseñanza de la literatura en educación infantil y primaria

Francesc Rodrigo-Segura y Josep Ballester-Roca

ASPECTOS MEJORAR

1.- Aparecen quejas en grupos concretos sobre problemas en el trabajo en equipo, la mayoría sobre las faltas injustificadas de algunos compañeros. 2.- Se propone mejorar la configuración de los equipos, puesto que no todos los equipos estaban equilibrados en cuanto a capacidad de trabajo.

CATEGORÍA 5: APRENDIZAJES DIDÁCTICOS

VALORACIÓN POSITIVA

1.- Los datos indican que el alumnado va cambiando su concepción de lo que es la literatura y de cómo se tiene que enseñar. También hay muchos datos que avalan la consideración del enfoque metodológico de la educación literaria como el mejor enfoque para enseñar literatura. 2.- Hay bastantes evidencias de cambios en la actitud hacia la asignatura: ha aumentado el valor y la importancia que se le da al hecho literario, se considera fundamental para obtener una educación y una competencia literaria elevada, se considera una herramienta interdisciplinar de gran valor pedagógico. 3.- Son conscientes de la importancia de adoptar el modelo de educación literaria para trabajar la literatura de una forma transversal, interdisciplinar y globalizadora y de la necesidad de trabajar en equipo.

CATEGORÍA 8: EVALUACIÓN

VALORACIÓN POSITIVA

1.- Es general la valoración positiva del proyecto basada en las criterios de: a) UTILIDAD: buena herramienta para difundir los recursos y las producciones generadas, nos ha ayudado a conseguir un aprendizaje práctico y significativo. "Como educadora, he planteado en mi centro la posibilidad de crear una, pues es un recurso útil para el aula y para comunicarte con las familias". "Es útil para en el futuro crear una web en nuestra aula". "Hemos desarrollado la competencia digital". b) INTERÉS: es muy interesante la creación de web, gracias a ella, podemos hacer llegar a otros compañeros y a profesionales de la educación los recursos que hemos creado, permite difundir materiales y recursos relacionados con la literatura y crear nuevos. C) INNOVACIÓN: ha sido el proyecto más creativo e innovador que hemos hecho hasta ahora, es muy interesante y enriquecedor, además de innovador, actividad significativa...

ASPECTOS A MEJORAR

1.- En los comentarios cualitativos aparece la queja que el trabajo del proyecto es excesiva y la propuesta general es aumentar su valor (+ del $25 \%)$.

Respecto al análisis cuantitativo de la experiencia, se presentan los resultados de las dos muestras realizadas: la encuesta de valoración del proyecto y el análisis de los resultados académicos de dos grupos de $2^{\circ}$ curso del Grado de Educación Infantil. 
Un proyecto integrado para la formación de los futuros maestros: elaboración de páginas web para la enseñanza de la literatura en educación infantil y primaria Francesc Rodrigo-Segura y Josep Ballester-Roca

Tabla 3

Encuesta de valoración del proyecto. (22 encuestas de los 30 alumnos del Grado de Maestro/a de Educación Infantil.

1.- En la Guía de la Asignatura aparecen de manera clara los obje- $\quad 90 \quad 4.1$ tivos, la metodología, los criterios e instrumentos de evaluación del proyecto.

2.- ¿Se ha explicado con detenimiento las fases del proyecto y las $\quad \begin{array}{lll}97 & 4.4\end{array}$ actividades a realizar?

3.- ¿Habéis dispuesto de los recursos y materiales suficientes para $\quad 86 \quad 3.9$ realizar el trabajo?

4.- ¿La ayuda de los estudiantes del CFGS en la realización de la $\quad \begin{array}{ll}46 & 2.1\end{array}$ web ha sido valiosa?

4.- ¿Consideráis que la combinación entre teoría y práctica ha sido 914.1 la adecuada?

5.- ¿Teníais clara la finalidad y la naturaleza del proyecto? $\quad 1004.5$

6.- ¿Tiene relación el proyecto con los objetivos de la asignatura y $\quad 90 \quad 4.1$ con el modelo de Formación Literaria que se propone?

7.- ¿Con la realización del proyecto habéis ampliado vuestra com- $96 \quad 4.3$

petencia oral y escrita en catalán?

8.- Hemos evolucionado positivamente en la capacidad de trabajo 894.0

en equipo.

9.- Hemos profundizado en el dominio de las TIC.

11.- ¿Hemos elaborado una muestra amplia y variada de textos de $94 \quad 4.2$

diversos géneros?

12.- Ha habido una buena coordinación de los equipos por parte $\quad 89 \quad 4.0$

del profesorado.

13.- El profesorado supervisa el trabajo individual y de los grupos $\quad 90 \quad 4.1$ en el aula.

13.- ¿Se han atendido correctamente a las dudas individuales o del $\quad 90 \quad 4.1$ grupo en clase o en horario de atención?

14.- ¿Se ha podido participar y tomar decisiones de manera autó- $\quad 98 \quad 4.5$

noma en la realización proyecto?

15.- Las actividades realizadas en clase, a lo largo del semestre, $\quad 98 \quad 4.5$

¿han sido útiles para vuestro futuro profesional?

17.- ¿Las actividades de presentación y de difusión del proyecto $\quad 93 \quad 4.2$

han sido adecuadas?

16.- ¿Están claros y son adecuados los criterios de evaluación del $\quad 95 \quad 4.3$

proyecto?

19.- ¿Existen suficientes instrumentos para evaluar el proyecto de $\quad 88 \quad 4.0$ creación de páginas web?

20.- ¿He podido participar la evaluación del proyecto?

$88 \quad 4.0$ 
De los resultados de la encuesta de valoración del proyecto cabe destacar que, a nivel global, los resultados son muy satisfactorios. De las 20 preguntas planteadas, 18 obtienen una puntuación de 4 o superior al 4 (en una escala del 1 al 5). El alumnado manifiesta estar bastante o muy de acuerdo con lo que se plantea en cada ítem. Destacan positivamente, con una puntuación de 4.5, los criterios de finalidad y naturaleza del proyecto, la participación y autonomía en la realización proyecto y la utilidad futura de las actividades realizadas. Estos resultados refuerzan las conclusiones cualitativas ya que en ambos casos se señalan como elementos mejor valorados los mismos criterios: el interés, la autonomía en su realización y la utilidad futura para la docencia. También hay coincidencia en los aspectos a mejora: la ayuda por parte de los estudiantes del CF de informática y los recursos y materiales necesarios para la realización del trabajo.

A continuación se presentan los resultados académicos de la asignatura de Formación Literaria en el aula de infantil5:

Tabla 4

Resultados del grupo $2^{\circ}$ A del Grado de Educación Infantil

\begin{tabular}{lcccc}
\hline 47 alumnos & METODOLOGIA $1(\sin$ P.I.) & METODOLOGIA 2 (con P.I.) \\
\hline INSUFICIENTE & 13 & $27.6 \%$ & 8 & $17.0 \%$ \\
SUFICIENTE & 14 & $29.8 \%$ & 5 & $10.6 \%$ \\
BIEN & 10 & $21.2 \%$ & 20 & $42.5 \%$ \\
NOTABLE & 9 & $19.1 \%$ & 11 & $23.4 \%$ \\
SOBRESALIENTE & 1 & $2.1 \%$ & 3 & $6.5 \%$ \\
\hline
\end{tabular}

Tabla 5

Resultados del grupo $2^{\circ} \mathrm{B}$ del Grado de Educación Infantil

\begin{tabular}{llllc}
\hline 34 alumnos & \multicolumn{1}{l}{ METODOLOGIA 1 (sin P.I.) } & \multicolumn{2}{l}{ METODOLOGIA 2 (con P.I.) } \\
\hline INSUFICIENTE & 6 & $17.6 \%$ & 1 & $2.9 \%$ \\
SUFICIENTE & 3 & $8.8 \%$ & 3 & $8.8 \%$ \\
BIEN & 11 & $32.4 \%$ & 10 & $29.4 \%$ \\
NOTABLE & 11 & $32.4 \%$ & 12 & $35.3 \%$ \\
SOBRESALIENTEE & 3 & $8.8 \%$ & 8 & $23.5 \%$ \\
\hline
\end{tabular}

5 Para establecer esta comparativa se ha utilizado el programa SPPS para realizar los cálculos estadísticos. Primero hemos realizado la Prueba de Kolmogorov-Smirnov para comprobar la normalidad de la muestra y el resultado es que la muestra es normal. A continuación, se han comparado las medias con la Prueba t-student para obtener los resultados. 
La comparación de los resultados se ha realizado comparando dos metodologías

a) El valor real de la nota final que contempla como instrumento de evaluación el Proyecto integrado, con un porcentaje del 25\% de la nota final.

b) El valor estimado de las notas finales sin tener en cuenta el porcentaje del proyecto.

Así pues, los resultados muestran que:

1. Sí hay un efecto significativo de la metodología: la metodología con proyecto funciona mejor que la metodología sin proyecto en los dos grupos analizados, tal y como demuestran los mejores resultados obtenidos.

2. El grupo de $2^{\circ} \mathrm{B}$ obtiene mejores resultados que el de $2^{\circ} \mathrm{A}$ en ambas metodologías.

Tabla 6

Ejemplos de páginas web

\begin{tabular}{|c|c|}
\hline $\begin{array}{l}\text { PROYECTOS } 2^{\circ} \text { GRADO EDUCA- } \\
\text { CIÓN INFANTIL }\end{array}$ & $\begin{array}{l}\text { PROYECTOS } 2^{\circ} \text { GRADO EDUCA- } \\
\text { CIÓN PRIMARIA }\end{array}$ \\
\hline $\begin{array}{l}\text { GRUPO TRENCACLOSQUES (Rom- } \\
\text { pecabezas) } \\
\text { Trencaclosques } \\
\text { http://grup-infantil.wix.com/trenca- } \\
\text { closques-aula } \\
\text { https://es-la.facebook.com/Aula-Tren- } \\
\text { caclosques-1430913763836181/ }\end{array}$ & $\begin{array}{l}\text { GRUPO PLASTIDECORS } \\
\text { http://www.wix.com/cris_lopez_92/ } \\
\text { plastidecorsflorida }\end{array}$ \\
\hline \multirow[t]{2}{*}{$\begin{array}{l}\text { GRUPO EUROPA } \\
\text { http://www.wix.com/somiemdiversi- } \\
\text { tat/europa } \\
\text { http://somiemdiversitat.blogspot.com/ } \\
\text { search/label/\%22L\%27aventura\%20 } \\
\text { de\%20Laura\%22 }\end{array}$} & $\begin{array}{l}\text { GRUPO LAS PELUSAS LITERARIAS } \\
\text { Ejemplo de la memoria escrita del } \\
\text { proyecto: } \\
\text { https://es.slideshare.net/Raquelpelu- } \\
\text { ses/formaci-tot-junt-este }\end{array}$ \\
\hline & $\begin{array}{l}\text { GRUPO ARC DE SANT MARTÍ (Arco } \\
\text { Iris) } \\
\text { http://grupprojecte555.wixsite.com/ } \\
\text { diversitatirecursos }\end{array}$ \\
\hline
\end{tabular}




\section{Conclusiones e implicaciones}

Una vez realizada la investigación, como conclusión global se considera que la respuesta a la hipótesis de partida es positiva, pues el proyecto sí que cumple con los objetivos formulados en su inicio, ya que el diseño de páginas web ha permitido adquirir el conjunto de aprendizajes (conceptuales, procedimentales y actitudinales) que se precisan para realizar un aprendizaje significativo de la literatura (Reyzábal y Tenorio, 1992, 2004). Además, ha contribuido al desarrollo práctico de las competencias específicas de la asignatura de formación literaria y de las competencias profesionales de trabajo en equipo, innovación y creatividad y dominio de las TIC. Las razones de esta aseveración son las siguientes:

En primer lugar cabe destacar que los resultados obtenidos han sido altamente satisfactorios tanto cuantitativamente (por las calificaciones obtenidas), como cualitativamente, por el alto nivel de satisfacción en las valoraciones del alumnado.

En segundo lugar se destaca que esta metodología contribuye a la creación de nuevos dispositivos de enseñanza, en la línea apuntada por Dolz (2009), para construir y/o a adaptar secuencias didácticas que mejoren la intervención en el aula. Además, como futuros maestros, experimentan una metodología que después podrán usar en sus clases y por lo tanto están desarrollando su competencia didáctica, ya que participan en el diseño de nuevos dispositivos de aprendizaje adaptados a las nuevas exigencias del sistema didáctico de la escuela actual. Por ello, los proyectos se convierten en dispositivos muy útiles para el aprendizaje de la lectura, la expresión oral y escrita y la enseñanza de la literatura. Además, en el caso de los estudiantes de magisterio, permiten establecer una relación directa entre los contenidos teóricos y la praxis educativa al utilizar recursos (como las TIC aplicadas a la LIJ) que contribuyen a mejorar su formación literaria y su preparación didáctica. En esta línea se valora muy positivamente el hecho que la realización de los proyectos contribuye de manera eficaz al reto de desarrollar la competencia digital de los futuros formadores puesto que esta capacidad se considera un eje fundamental de nuestra sociedad, tal y como destacan Grande-de-Prado, Cañón-Rodríguez, y Cantón-Mayo (2016).

Respecto a los objetivos y los aprendizajes específicos lingüísticos y literarios, se constata que ha habido un elevado grado de conocimiento y desarrollo de los contenidos del currículum de Formación Literaria y 
que éste se ha realizado de manera práctica. Se ha trabajado con profundidad y de manera globalizada la variedad discursiva textual y las producciones literarias y académicas (expositivas o argumentativas), reunidas en un producto final que permite presentar el trabajo colectivo al resto de compañeros y a la comunidad educativa.

En el planteamiento del proyecto y el desarrollo de las clases se ha insistido mucho en la calidad de las producciones y por ello la publicación de los trabajos en la web implicaba un esfuerzo previo de selección y de corrección. Uno de los criterios de evaluación era la riqueza, variedad y corrección en el contenido y en las formas de presentación mediante diversas herramientas informáticas. También se constata la integración en el proyecto de los diversos aprendizajes de la asignatura: los específicos de formación literaria (técnicas y recursos para la animación literaria, lectura de libros y artículos sobre la materia), los lingüísticos y textuales (amplia y variada producción textual) y los competenciales (dominio de las TIC, trabajo en equipo, creatividad e innovación). Por otro lado, se ha podido comprobar la trascendencia social del trabajo generado puesto que se han compartido productos, recursos y experiencias, con el objetivo que todos puedan enriquecerse con los productos realizados.

Respecto a los resultados, el profesorado participante concluye que han sido satisfactorios y destaca que se ha realizado una experiencia real basada en el desarrollo de competencias profesionales y el trabajo entre iguales. Los propios estudiantes asesoran y forman a compañeros de titulaciones diferentes. Y así se han podido generar recursos de aplicabilidad inmediata (web/blog), consiguiendo un elevado grado de transferencia del saber adquirido. En los proyectos se han integrado diversas disciplinas consiguiendo la transferencia armónica de los aprendizajes desde el paradigma de la complejidad (integrando conocimientos de Formación Literaria, Sociología, Educación Especial, etc.). Otro aspecto fundamental de la experiencia ha sido la creación de un espacio de colaboración permanente entre los docentes que facilita la coordinación y permite solucionar las necesidades pedagógicas comunes.

Desde el punto de vista metodológico, cabe señalar que el diseño de las páginas web ha permitido utilizar las TIC en el aula como un recurso innovador e integrador del trabajo de diversas disciplinas. También se han utilizado metodologías diversas y se han generado dinámicas de trabajo prácticas y altamente motivadoras. Además, se ha producido un cambio en el rol de los docentes, que trabajan de manera interdiscipli- 
naria y cooperativa, abandonando el papel tradicional de transmisores de conocimiento y adquiriendo un nuevo papel como dinamizadores de procesos de aprendizaje.

Así pues, se puede considerar que los proyectos de lengua y literatura en el ámbito de la enseñanza universitaria se convierten en una forma de planificación didáctica eficaz y constituyen un instrumento válido para la observación y la investigación didáctica por parte del profesorado. Esta metodología permite llevar a las aulas los principios del paradigma de la educación literaria y contribuye a realizar un aprendizaje significativo de la literatura.

Finalmente, se concluye que esta investigación permite adecuar las prácticas de enseñanza y aprendizaje de la literatura, en el nivel universitario, a las necesidades del alumnado y a las exigencias de cambios en el sistema didáctico. Y consigue aportar algunos elementos de interés para futuras reflexiones que contribuyan a la innovación didáctica desde la práctica docente, la investigación y la reflexión posterior.

\section{Referencias}

Arnal, J., del Rincón, D. y Latorre, A. (1992). Investigación educativa: Fundamentos y Metodología, 245-263. Barcelona: Lapor.

Ballester, J. (1999). L'educació literària. València: Universitat de València, 2a ed. 2007.

Ballester, J. (2015). La formación lectora y literaria. Barcelona: Graó.

Ballester, J. e Ibarra, N. (2013a). La tentación diabólica de instruir. Reflexiones a propósito de la educación lectora y literaria. OCNOS, 10, 7-26.

Ballester, J. e Ibarra, N. (2013b). La literatura infantil y juvenil en la formación del maestro. Sección Monográfica sobre la LIJ, Lenguaje y Textos, 38, 11-18.

Ballester, J. e Ibarra, N. (2016). La educación lectora, literaria y el libro digital, Revista Chilena de Literatura, 94, 147-171.

Camps, A. (2012a). La investigación en didáctica de la lengua en la encrucijada de muchos caminos. Revista Iberoamericana de Educación, 59, 23-41.

Camps, A. (2012b). Intervenció, innovació i investigació. Una relació necessària per a les didàctiques. Lenguaje y Textos, 35, 107-114.

Cerrillo, P. (1990). Literatura Infantil y Universidad. En Cerrillo, P. \& García Padrino, J. (coords.) Literatura Infantil (pp. 11-19). Cuenca: Universidad de Castilla-La Mancha.

Cerrillo, P. (2007). Literatura Infantil y Juvenil y educación literaria. Barcelona: Octaedro.

Colomer, T. (2002). La lectura infantil y juvenil. En Millán, J. A. (coord.), La lectura en España. Informe 2002 (pp. 263-285). Madrid: Federación de Gremios de Editores de España. 
Un proyecto integrado para la formación de los futuros maestros: elaboración de páginas web para la enseñanza de la literatura en educación infantil y primaria

Francesc Rodrigo-Segura y Josep Ballester-Roca

Colomer, T. (2005). Andar entre libros: la lectura literaria en la escuela. México: Fondo de Cultura Económica.

Colomer, T. (2012). L'ensenyament de la literatura a l'ESO: primeres preguntes. Articles, $56,81-87$.

Deleuze, G. y Guattari, F. (2001). Rizoma. (C. Casillas y V.Navarro, Trads.). México, D. F.: qCoyoacán. (Trabajo original presentado en 1994). València: Pre-Textos. (7a. ed. 2010).

Delors, J. (1996). Educació: hi ha un tresor amagat dins. Informe a la UNESCO de la Comissió Internacional sobre I'Educació per al segle XXI, Centre UNESCO de Catalunya. Reuperado de http://www.unescocat.org/ca/recursos/publicacions/educaciohi-ha-un-tresor-amagat-a-dins

Desgagné, S. (1997). Le concept de recherche collaborative : I'idée d'un rapprochement entre chercheurs universitaires et praticiens enseignants.Érudit. Revue des sciences de l'éducation, 23 (2), 371-393. Recuperado de http://id.erudit.org/iderudit/031921 [Consulta septiembre 2017].

Dolz, J. (2009): Los cinco grandes retos de la formación del profesorado de lenguas. $V$ SICET. UCS: Universidade de Caxias do Sul. Brasil: Caxias do sul.

Engeström, Y. (1987). Learning by Expanding: An Activity-Theoretical Approach to Developmental Research. Helsinki: Orienta-Konsultit.

Freire, P. (1975). Pedagogía del oprimido. Madrid: Siglo XXI.

Grande-de-Prado, M.; Cañón-Rodríguez, R. y Cantón-Mayo, I (2016). Competencia digital y tratamiento de la información en futuros maestros de Primaria. Educatio Siglo XXI, 34 (3), 101-118.

Guba, E, C y Lincoln, Y. S. (2000): Paradigmas en competencia en la investigación cualitativa. En C.A. Denman i J.A. Haro (eds), Por los rincones, Antología de métodos cualitativos en la investigación social (pp. 113-145).. Hermosillo, Sonora, Mèxico: El Colegio de Sonora.

Latorre, A. 2003. La investigación-acción. Conocer y cambiar la práctica educativa. Barcelona: Graó.

Lewin, Kurt 1946. Action research and minority problems. Journal of Social Issues, 2 (4): 34-46.

Manresa, M.; Duran, T. y Ramada, L. (2012). Les TIC en les seqüències didàctiques de Ilengua i literatura. Articles, 57, 38-48.

Margallo, A. (2005). La enseñanza literaria a través de proyectos. (Tesis doctoral). Universitat Autònoma de Barcelona, Barcelona. Recuperado de http://diposit.ub.edu/dspace/bitstream/2445/28923/1/ridell1.pdf

Margallo, A. (2012a). La educación literaria en los proyectos de trabajo. Revista Iberoamericana de educación, 59, 139-156.

Margallo, A. (2012b). La formació de lectors literaris a través de les seqüències didàctiques en forma de projecte, Articles, 57, 22-35.

Mendoza, A. (1998). Marco para una Didáctica de la Lengua y de la Literatura en la formación de profesores, Didáctica. Llengua y literatura, 10, 233-269.

Mendoza, A. (2004). La educación literaria. Bases para la formación de la competencia lecto-literaria, Málaga: Aljibe. 
Un proyecto integrado para la formación de los futuros maestros: elaboración de páginas web para la enseñanza de la literatura en educación infantil y primaria

Francesc Rodrigo-Segura y Josep Ballester-Roca

Mendoza, A. (2011). La Investigación en Didáctica de las Primeras Lenguas. Educatio Siglo XXI, 29 (1), 31-80.

Morin, E. (1999). Los siete saberes necesarios para la educación del futuro. Paris: UNESCO.

-(2006). El Método V. la humanidad de la humanidad. La identidad humana. Madrid: Cátedra. $2^{\mathrm{a}}$ ed.

Reyzábal, Mª V. \& Tenorio, P. (1992). El aprendizaje significativo de la literatura, Madrid: La Muralla, $4^{\mathrm{a}}$ ed. 2004.

Rodrigo, F. (2012). L'educació literària: una proposta de treball per projectes. Trabajo Fin del Máster en Investigación en Didácticas Específicas. Universidad de Valencia.

Rodrigo, F. (2016). La formació literària i la metodologia de projectes de treball en batxillerat $i$ en els graus de mestre/a d'Educació Infantil i Primària (Tesis Doctoral). Universidad de Valencia, Valencia. Recuperado de http://roderic.uv.es/bitstream/item/59505/TESIS\%20FRANCESC\%20RODRIGO\%20-\%20DEFINITIVA. pdf? sequence $=1$ \&isAllowed $=y$

Rodrigo, y otros (2012): Desenvolupament d'un model educatiu centrat en les competències professionals i el treball per projectes en els graus d'educació de Florida Universitària. Revista Congreso Internacional de Docencia Universitaria e Innovación (CIDUI). Barcelona: Universitat Pompeu Fabra. Disponible en: http://www.cidui.org/ revista-cidui12/index.php/cidui/issue/view/

Stenhouse, Lawrence 1991: Investigación y desarrollo del currículo. Madrid: Morata.

Zayas, F. (2011). Educación Literaria en la era digital. Monográfico de Educación Literaria y TIC. Leer.es. Madrid: MEC. 
